\title{
Turismo disruptivo: rutas turísticas inteligentes
}

\section{Disruptive tourism: smart tourist routes}

Recibido: 15/06/2020

Aceptado: $15 / 07 / 2020$

\author{
Santiago Patricio Pulla Pesantez ${ }^{1}$, Adriana Lucila Ortega Echeverria², Kleber Patricio \\ Castro Pacheco ${ }^{3}$
}

1. Coordinador de la carrera de turismo del Instituto Tecnológico Superior Particular Sudamericano, Cuenca - Ecuador.

Email: sapulla@sudamericano.edu.ec ORCID: https://orcid.org/0000-0001-7212-238X

2. Docente del Instituto Tecnológico Superior Particular Sudamericano, Cuenca - Ecuador.

Email: alortega2@sudamericano.edu.ec ORCID: https://orcid.org/0000-0002-2800-7744

3. PhD en Ciencias económicas mención Turismo, Consultor Sénior, Newark, NJ USA.

Email: patricastro@yahoo.com ORCID: https://orcid.org/0000-0002-7714-1501

Para Citar: Pulla Pesantez, S. P., Ortega Echeverria, A. L., \& Castro Pacheco, K. P. (2020). Turismo disruptivo: rutas turísticas inteligentes. Revista Publicando, 7(25), 160-169. Recuperado a partir de https://revistapublicando.org/revista/index.php/crv/article/view/2100.

Resumen: La presente investigación tiene como propósito sustentar la posible utilización de las rutas y circuitos turísticos de manera inteligente, para ello se propone consolidar la utilidad del territorio turístico inteligente, la visualización de datos, el acceso de los datos en las infraestructuras de conectividad y sensorización dentro de un modelo de Destino Turístico Inteligente. El objetivo es entender la evolución de las Tecnologías de la Información y Comunicación dentro de un espacio determinado que genere información en tiempo real de los atractivos turísticos naturales y culturales, los accesos a los mismos y las facilidades en el momento de realizar la actividad de ocio y recreación. El modelo de ruta que se plantea considera el trascender en la actual realidad del sector turismo, que se encuentre abierto a trabajar de forma multidisciplinar con otros sectores que propicien el uso sustentable de los recursos culturales y naturales a través del Internet de las Cosas.

Palabras clave: Territorio inteligente, rutas, circuitos, visualización de datos, Destinos Turísticos Inteligentes.

\begin{abstract}
The purpose of this research is to support the possible use of tourist routes and circuits in an intelligent way, for this purpose it is proposed to consolidate the usefulness of the intelligent tourist territory, data visualization, data access in connectivity infrastructures sensorization within a Smart Tourist Destination model. The objective is to understand the evolution of Information and Communication Technologies within a given space that generates real-time information on natural and cultural tourist attractions, access to them and facilities at the time of leisure activity. and recreation. The proposed route model considers transcending the current reality of the tourism sector, which is open to working in a multidisciplinary way with other sectors that promote the sustainable use of cultural and natural resources through the Internet of Things.
\end{abstract}

Keywords: Smart territory, routes, circuits, data visualization, Smart Tourist Destinations. 


\section{INTRODUCCIÓN}

$\mathrm{S}$ e define a la ruta turística como el espacio físico de la geografía que contiene diferentes atractivos turísticos naturales y culturales, los cuales por medio de los accesos y las facilidades sirven para la realización de diferentes actividades de tipo turístico y/o recreativo, para el disfrute de los turistas y/o visitantes en un lugar (Castro, 2020). En palabras de López-Guzmán y Sánchez (2008) es el conjunto de actividades y atractivos a lo largo de un área o de uno o varios territorios, debe comprender diversos elementos turísticos y las facilidades de acceso a los mismos.

Este enfoque sirve de base para reconocer como ejemplo una de las rutas más emblemáticas y que es Patrimonio Cultural de la Humanidad, el camino de Santiago de Compostela (Blanco, 1995). La condicionante primaria y principal para definir y delimitar una ruta es el espacio geográfico físico, el cual tiene varias formas de entenderlas desde el punto de vista de la estructuración del espacio turístico, que puede ser homogéneo o heterogéneo (Boullon, 1995).

Las primeras rutas a las que se hace memoria y se asocian a la actividad del turismo son las utilizadas en las guerras santas en época de las cruzadas, puesto que fueron determinados espacios en función de las necesidades de los visitantes que acudían en peregrinación.

Se reconoce al camino de Santiago de Compostela como un lugar sagrado de peregrinación desde un ámbito cronológico con orígenes que remontan a los tiempos de la primera difusión del cristianismo de la península durante la dominación Romana. La historia del camino de Compostela remota desde los siglos XI Y XII con el eje rural y agrícola, favoreciendo al crecimiento del comercio y la industria de los habitantes cercanos a la misma.

En 1124 se decretó la Paz de Dios entre territorios de Francia, Rosellón, Cataluña y Braga, de esta forma la relación entre la peregrinación y los movimientos de renovación de la vida social y política de la época cambiaron para convertirse en el clima de paz y seguridad. Cabe destacar que el peregrino contaba con información sobre las condiciones del viaje. Allí se inició el principal texto conocido, a este respecto como la Guía del Peregrino atribuida a Aimerico Picaud escrita en 1160 , con estas consideraciones se puede establecer el ejemplo de un itinerario utilizado en una ruta religiosa (Blanco, 1995).

En las actuales circunstancias de entornos dinámicos y cambiantes, se están configurando los denominados territorios inteligentes, en base a la capacidad de utilización de la tecnología en la vida diaria y normal del ser humano, por el aprovechamiento de dispositivos móviles y aplicaciones digitales que determinan las acciones a seguir en un tiempo y espacio delimitado, como es el uso de GPS (Geographical Posictional System) que es un instrumento de uso diario para cubrir y abarcar distintos puntos a conectar a través del uso de los medios de locomoción como son los autos, motos, bicicletas y por el ser humano.

Es importante resaltar que esta publicación forma parte del proyecto de investigación Turismo Disruptivo: Rutas Turísticas Inteligentes, desarrollado a través del financiamiento del Instituto Tecnológico Superior Sudamericano como contribución para el impulso del Turismo como actividad económica por excelencia en el Ecuador.

\section{DESARROLLO}

\section{LOS TERRITORIOS INTELIGENTES}

El desarrollo a nivel de las Tecnologías de la Información y Comunicación (TIC) ha propiciado que las personas puedan acceder a una cantidad infinita de contenidos digitales además de la aparición de dispositivos portátiles en donde convergen infinidad de aplicaciones y contenidos como cultura, ocio, turismo, educación, salud, etc., (CabezueloLorenzo et al., 2016); la evolución de la tecnología representa un importante factor que ha transformado la conducta del consumidor, eso ha obligado a las empresas a adaptarse a esta evolución no siendo ajena a este hecho, la industria turística.

Las TIC generan ventajas a nivel de competitividad, automatización de procesos, disminución de errores, etc., la importancia de una comunicación rápida el ahorro de costos y reducción del tiempo en los procesos es esencial en la actividad turística, procesos confiables que permitan la promoción y compra de productos y servicios dentro de la industria, las imágenes, vídeos, la transmisión de emociones 
son la base para generar la motivación en el consumidor que busca ocio (Valles, 1999).

A partir de la transformación de sentidos y emociones en el ser humano se establece la creación del concepto de un territorio inteligente, que en su proceso amplio se consolida en las denominadas ciudades inteligentes o "Smart cities", término que se empieza a utilizar aproximadamente en el año 2008, como un ideal de incorporación de tecnologías digitales dentro de la ciudad, en donde sólo un pequeño grupo de empresas pioneras había comenzado a utilizar este término; se dice también que en paralelo surgían algunas propuestas para aplicar la tecnología a modelos urbanos de desarrollo sostenible (Fernández, 2016).

Según, Boyd Cohen:

La primera ciudad, Smart City surge dada la necesidad de orientar la vida hacia la sostenibilidad y sustentabilidad, con ello se pone énfasis en la innovación de las tecnologías, para mejorar el estilo de vida y las acciones para preservar los bienes naturales y culturales para el futuro, reduciendo emisiones dañinas $(2016,46)$.

La evolución de la idea de una ciudad inteligente desemboca en el concepto de "destino inteligente", sin embargo, una serie de transformaciones estructurales en el sector turístico, justifican esta necesidad de un cambio en la gestión; el concepto de una "Smart destination” según Segittur, (2017, p.24):

Un destino turístico innovador, consolidado sobre una infraestructura tecnológica de vanguardia, que garantiza el desarrollo sostenible del territorio turístico, que promueve la accesibilidad universal, que facilita la interacción e integración del visitante con el entorno e incrementa la calidad de su experiencia en el destino y mejora la calidad de vida de los residentes.

El mercado turístico y el dinamismo en cuanto a las tendencias de la demanda que generan los turistas, que cambian a medida que avanza el tiempo los hábitos de consumo, y propician la generación de productos diferentes, a partir de esto nace una propuesta de generar un modelo de ruta, que responda a esta nueva necesidad y que se podría denominar como una ruta turística inteligente.
En los últimos años, el concepto de territorio inteligente ha tenido un creciente calado entre expertos y agentes dedicados al estudio del desarrollo sostenible. Desde la perspectiva del urbanismo y la arquitectura, destaca la definición realizada por Alfonso Vegara y Juan Luis de las Rivas (2014), los cuales son los autores que, de manera más explícita, acuñan el concepto de territorio inteligente en los siguientes términos:

Los territorios inteligentes son aquellos territorios innovadores, capaces de construir sus propias ventajas competitivas en relación con su entorno, en el marco de un mundo complejo, global e interrelacionado. Asimismo, los territorios inteligentes persiguen un equilibrio entre los aspectos de competitividad económica, cohesión social y sostenibilidad.

En definitiva, los territorios inteligentes son aquellos capaces de dotarse de proyectos de ciudad, de lograr el equilibrio justo, de descubrir su singularidad y de construir sus propias ventajas competitivas en un marco global. Cualquier territorio, al margen de su tamaño y nivel de infraestructuras, puede ser un territorio inteligente.

Se debe recalcar que la definición del territorio inteligente al ser una nueva acepción surge como un nuevo concepto teórico de las confluencias de distintas disciplinas científicas relacionadas, de manera principal en el urbanismo, la arquitectura, el patrimonio cultural, el medio ambiente, la economía de la innovación y el desarrollo regional, pero sobre todo se aplica el enfoque de la multidisciplinariedad de las ciencias diversas, que generan sinergias varias con el propósito de consolidar para la actividad turística un modelo que debe ser explotado en las actuales circunstancias como es el de los Destinos Turísticos Inteligentes, que son el presente en el mundo (Castro, 2020).

Esta capacidad multidisciplinar y al mismo tiempo de integración de distintas áreas, enfocadas desde el análisis del desarrollo de las infraestructuras en conectividad y sensorización, la idea del territorio inteligente toma especial relevancia si se tiene en cuenta que, hasta el presente, el ámbito de estudio del desarrollo económico y social es abordado desde diferentes perspectivas de análisis lo que hace necesario repensar la actual forma de enfoque de la economía, que debe ser considerada de manera inteligente dentro de un sistema holístico que una atractivos, accesos y 
facilidades, con la gestión local, la disponibilidad de un precio y por supuesto tener calidad; así se confirmaría la concepción del producto en un destino, donde las rutas y circuitos forman parte de la actividad.

\section{La visualización de datos como herramienta de consolidación de las rutas inteligentes}

El ejemplo más importante es el trabajo realizado de la visualización de datos del inventario de atractivos turísticos, que genera una alternativa real de conceptualización de la actividad desde la perspectiva de innovación tecnológica y de cambio en el enfoque tradicional del concepto del turismo (Castro, 2010).

Una de las formas de consolidar y generar ciencia turística es por medio de la unión y fusión de las diversas disciplinas, con la idea de generar sinergias positivas. Es el caso de la investigación que engloba el registro y la evaluación de los elementos del patrimonio turístico (PT), enlazados a la visualización de datos (open data) que son creados por gráficos de computadoras, que generan destrezas que se refieren a la más avanzada utilización tecnológica, permitiendo de esta manera una interpretación sensorial, mediante la representación, el modelado y la percepción de elementos sólidos, de grandes superficies, propiedades en animaciones en 2 y 3 D. Estas aplicaciones informáticas (bases de datos, procesadores de gráficos y palabras, entre otras) no son aplicadas a la actividad turística, por lo que no existe mayor información para comparar y validar de manera documentada, aplicada a dos ámbitos: rural y urbano.

La visualización de datos es una disciplina transversal que utiliza el inmenso poder de comunicación de las imágenes para explicar de manera comprensible las relaciones de significado, causa y dependencia que se pueden encontrar entre las grandes masas abstractas de información que generan los procesos científicos y sociales. Se puede manifestar que en el campo del turismo no ha sido del todo explorado, ya que no existen mayores aplicaciones, de esta forma se convierte en una experimentación tecnológica científica dentro de la planificación y gestión turística, debido a su forma y el ordenamiento de los atractivos naturales y culturales; donde son aplicados con la idea de poder determinar sus posibles usos (Castro, 2010).
El inventario, jerarquización y categorización de los atractivos turísticos, constituyen los pasos más importantes en el proceso de evaluación del potencial turístico de una región, por ende, determinan de forma particular la vocación real turística del territorio en el cual se intervendrá; dependiendo del ámbito de su aplicación se convierte en la base de la estructuración del espacio turístico de una región, país, zona, departamento, municipio o comunidad. De allí la importancia de considerar el inventario turístico para generar las rutas y circuitos.

Por lo tanto, se puede entender, que la única manera de confirmar que un territorio es turístico o no, y que justifique determinadas inversiones con los riesgos de ser malas, es a través de una evaluación rigurosa que tenga en cuenta los atractivos del territorio, la oferta, la demanda, la competencia y las tendencias del mercado, de esta manera la evaluación precisa del territorio constituye una excelente base de adopción de decisiones para los organismos de desarrollo.

Valorar el territorio turístico conlleva tres fases fundamentales:

1. Análisis de la situación turística existente, fase en la que se examinan los recursos, la oferta, la demanda, la competencia y las tendencias del mercado;

2. Diagnóstico que, comparando los resultados del análisis de la situación, permitirá identificar los puntos fuertes y débiles del territorio, las oportunidades y los riesgos;

3. Estrategias: si existe el potencial, definir la estrategia a seguir para el desarrollo turístico en el territorio (Zimmer y Grassmann, 1996).

Estos tres ciclos implican la recolección, tratamiento y análisis de informaciones internas y externas al territorio con características fuertes de brindar una oferta turística.

\section{Implementación de la Realidad Aumentada}

La aparición de Internet ha provocado la revolución en el mundo, en las empresas, en la población y el turismo, ha generado un cambio en el consumidor y en los canales de venta, esta forma de comercializar reduce la participación de terceros en el proceso y mejora la competitividad, así 
también, la aparición de un tipo de consumidor vinculado al mundo digital que dispone de su propio teléfono inteligente y que decide exactamente lo que desea. Los agentes del sector turístico han debido adaptarse a las nuevas tecnologías, a la innovación y transformación de sus productos tradicionales. La adaptación de estos avances tecnológicos otorga mayor valor a los productos provocando sensaciones, emociones y experiencias (Fernández y Cuadrado, 2014).

El éxito en la gestión de un destino turístico está en la habilidad de detectar y reconocer todos los cambios que se producen en el entorno, su dirección y la forma en que éstos afectan (Aguayo et al, 2010). La calidad de la oferta turística y la facilidad de promocionar adecuadamente un destino en donde toda la información este integrada en un sistema, es una de las razones por las cuales la adaptación a las nuevas tecnologías es necesaria dentro de la actividad.

La aparición del neo tipo de turista, que se encuentra en la búsqueda de experiencias recientes, hace que tanto él, como el destino utilicen herramientas recientes que faciliten el procedimiento de selección de aquello que busca, para ello la percepción de información gráfica y textual a disposición móvil es casi primordial, así también la posibilidad de leer las opiniones de otras personas en las que se ve reflejado, las opciones para acceder a dicha información cada vez son más numerosas, siendo una de ellas la realidad aumentada que se encuentra en auge (Fernández y Cuadrado, 2014).

El turista extranjero tiene una edad promedio de 36 años de género masculino, soltero y de instrucción superior, para planificar su viaje utiliza mayoritariamente Internet, puesto que ha pasado gran parte de su vida en una era en la que está rodeado de la tecnología, requiere servicios adecuados y personalizados, así también le gusta informarse acerca del destino al que llega; por tanto el turista que visita Ecuador no es ajeno a la necesidad de generar propuestas que faciliten el acceso a la información y proporcionen una experiencia deseable para el turista.

En cuanto, a las experiencias, la realidad aumentada es una herramienta de innovación que pretende fusionar el mundo real con el mundo virtual, en otras palabras, muestra contenidos en tiempo real a través de un dispositivo, la diferencia entre la realidad aumentada y la realidad virtual debe ser considerada, esta última muestra elementos incorporados de manera virtual, mientras que la realidad aumentada muestra elementos reales dentro de un espacio.

Aunque es una tecnología que se ha trabajado desde los años 90, únicamente en los últimos años es que ha sido posible la adaptación de esta tecnología a un dispositivo móvil; la incorporación de este tipo de tecnología a la actividad turística no sólo es útil para la promoción previa del destino, sino durante la visita, debido a que se puede incluir información multimedia que complemente la visita del turista en tiempo real.

La utilización de realidad aumentada incorporada a las rutas turísticas es una tecnología que permite obtener información desde la web, es de fácil acceso y utilización, así también las posibilidades de información para el turista son infinitas, esto se traduce como un proceso de mejora y un paso más hacia la inteligencia de un destino (Fernández y Cuadrado, 2014)

\section{Aplicación del modelo de gestión del Destino Turístico Inteligente}

El concepto destino turístico inteligente no puede ser pensado solamente como la aplicación del paradigma de ciudad inteligente (Smart City) al sector turístico, como ha sucedido habitualmente. Una serie de cambios estructurales en el sector turístico, que confluyen con la consolidación del paradigma de ciudad inteligente, justifican la necesidad de nuevos enfoques en la gestión de los destinos turísticos (Comunidad Valenciana, 2014, p.36).

Por tanto, es necesario revisar algunas definiciones del concepto; entre ellas se destacan: de acuerdo con el Consejo de Ministros de España (Plan Nacional Integral de Turismo 2012-2015, p.71) "El DTI, es un espacio innovador, accesible para todos, consolidado sobre tecnología de vanguardia, que garantiza el desarrollo sostenible del territorio, facilita la interacción e integración del visitante con el entorno e incrementa la calidad de su visita en el destino". Esta definición se basa en que la innovación es un espacio importante y funciona como eje central de todas las propuestas.

En palabras de SEGITTUR (2015, p.29) establece como destinos inteligentes los espacios innovadores, que utilizan tecnologías de vanguardia para impulsar el desarrollo 
sostenible en la gestión eficiente del recurso. Este enfoque consolida la propuesta anterior sobre una infraestructura tecnológica de vanguardia del territorio turístico, accesible para todos, facilite la interacción e integración del visitante con el entorno e incremente la calidad de su experiencia. Se trata de utilizar las TIC para promover la gestión eficiente, reducir el gasto y mejorar la calidad de vida de los ciudadanos.

Por su parte, el Libro Blanco de los Destinos Turísticos Inteligentes (2015, p.32), destaca la innovación y desarrollo de las TIC como base de los nuevos mecanismos para impulsar los DTI. La idea es crear servicios diferenciales y altamente competitivos, ganando en idoneidad $y$ rentabilidad, generar acciones que reconvirtieran las amenazas en oportunidades y permitan diferenciar el producto, luchar contra la estacionalidad, impulsar la diversificación y consolidar el éxito.

A partir de estos autores se pueden formular algunas concepciones sobre lo que es un DTI. Se destaca la definición de SEGITTUR, debido a su transcendencia en la actividad turística (la OMT utilizó dicha definición en la promoción del 1er Congreso Mundial OMT sobre Smart Destination, realizada del 15 al 17 de febrero de 2017, en Murcia, España): "Desde el enfoque holístico, el DTI implica nuevos modelos de gestión empresarial, nuevas formas de comunicación, la búsqueda del bienestar del cliente en un sentido amplio" (SEGITTUR 2017, p.17).

Son espacios innovadores que utilizan TIC para impulsar el desarrollo sostenible del territorio turístico, fomenta la innovación para una gestión eficiente de los recursos, accesible para todos, facilita la interacción e integración del visitante con el entorno y sus productos, reduce el gasto $y$ mejora la calidad de vida de los ciudadanos (Castro, Vásquez y Salgado, 2019).
La configuración de un DTI debe responder a las necesidades de cada destino y a los beneficios que pueda reportar, puesto que la sola adquisición y aplicación de tecnología a un destino no lo convierte en un DTI, en ese sentido, ésta debe ir acompañada de un proceso de cambio en todos los niveles (estrategia turística, mentalidad, cultura empresarial, capacidad de absorción de la innovación, generación de nuevos modelos de negocio, etc.).

No se puede considerar un DTI si utiliza o no las herramientas disponibles en beneficio de satisfacer el mercado, puesto que la tecnología es un medio no un fin, por lo que no deja de considerar cuestiones relacionadas con la sostenibilidad, la innovación o la accesibilidad. Buhalis y Amaranggana (2014), revisan la noción de DTI como un marco genérico que se compone de conceptos heredados de las Ciudades Inteligentes e incorporan a su base elementos como la competitividad, la sostenibilidad y la inclusión. Pretenden, de esta manera, proporcionar un marco holístico para los destinos turísticos inteligentes. No se trata, en fin, de hacer lo mismo con nuevas aplicaciones tecnológicas si no de revolucionar la gestión turística de acuerdo con las posibilidades tecnológicas y la capacidad de actuación local (Landslade et al., 2020).

Rutas inteligentes con la adaptación de herramientas (Global Position System, mapas, visualización de datos de los atractivos, etc.)

A partir del análisis de los diferentes formatos indicados en esta investigación, se plantea la posibilidad de que al unirlos en forma disciplinar, se establece una herramienta que consolida a la actividad turística y trascienda de un sistema no digital a uno de forma inteligente que se fortalezca de los diferentes aspectos para generar consistencia y fortalezas de cara a la capacidad infinita de manejar y trabajar en forma virtual. 


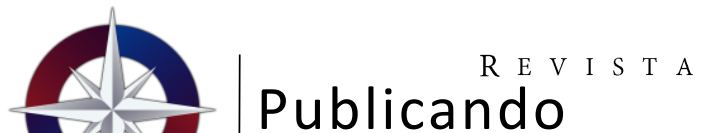

Figura 1. Rutas y corredores turísticos. Cuenca - Ricaurte - Cojitambo - Azogues.

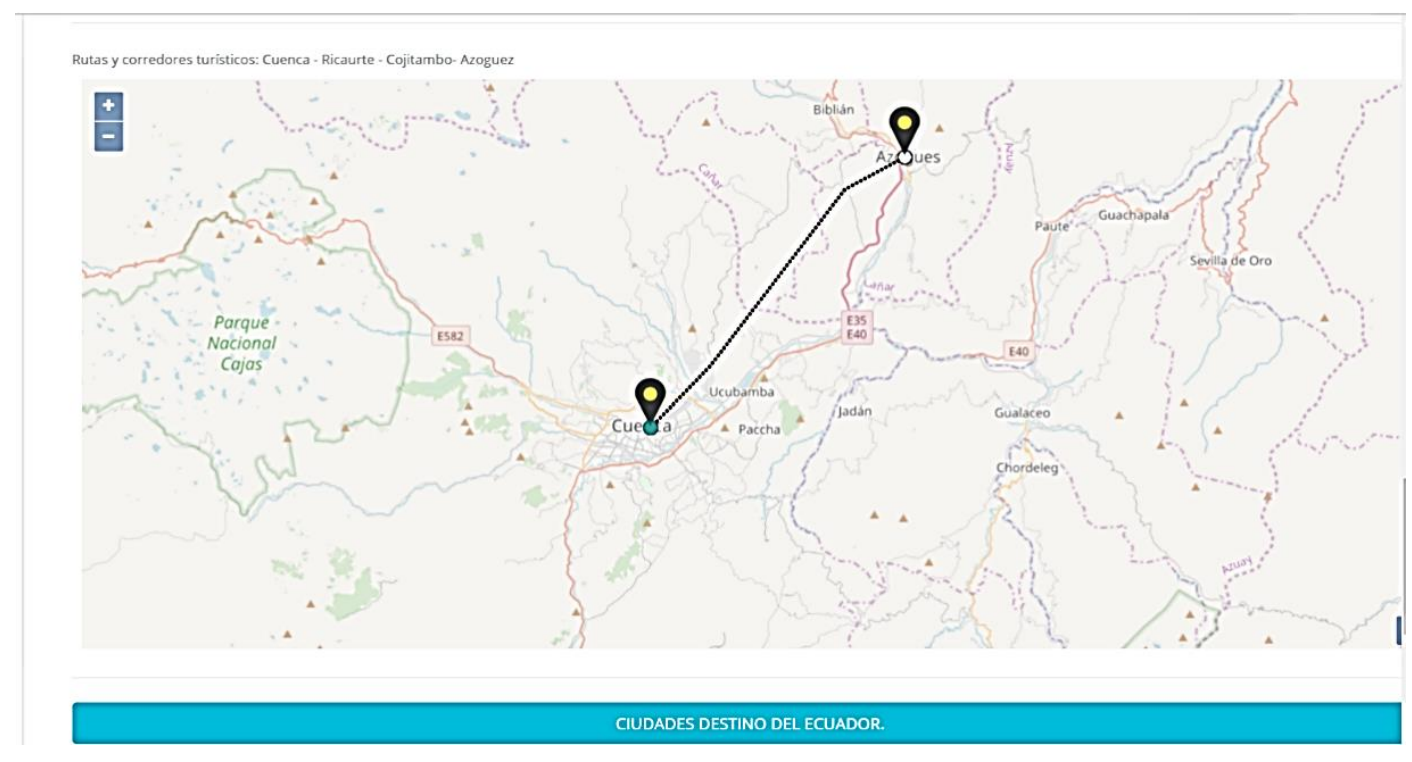

Fuente: Cuenca Destino Turístico Inteligente (2020).

Como se puede apreciar en la figura No.1, la ruta que se explora es desde Cuenca a Azogues, existe la capacidad de generar la misma de manera inteligente para consolidar el territorio inteligente del turismo, en forma amplia, holística e integral, que genere el uso de sinergias y enfoque capacidades de actuación con el fin de proponer un producto turístico y que se gestione como destino turístico final.

El procedimiento para levantar la información se hará en las siguientes actividades:

1. Levantamiento del inventario turístico activo (ITA) de atractivos turísticos naturales y culturales: es importante definir las capacidades reales y de jerarquía para determinar la calidad de la experiencia turística. Castro, 2016.

2. Análisis de los accesos: diferentes vías y caminos de conexión a atractivos, productos y destinos.

3. Análisis de las facilidades: capacidad instalada para dotación de actividades de turistas y/o visitantes en la ruta.
4. Generación de las rutas de acuerdo con los atractivos turísticos: turismo rural, turismo cultural, turismo religioso, turismo arqueológico, pueblos y caminos pintorescos, turismo gastronómico, etc.

5. Gestión local de la actividad: aplicación del modelo de gestión del Destino Turístico Inteligente en forma integral, donde se utilice visualización de datos, manejo de recursos, indicadores de gestión, territorio turístico inteligente, entre otras posibilidades.

6. Promoción y marketing digital: aplicación del programa de marketing digital integral.

Ese trabajo requiere de utilizar el sistema de Inventarios Turísticos Activos (naturales y culturales) que en un espacio se unen con los denominados accesos (de todo tipo e índole) para que junto a las facilidades (dotaciones del espacio para hacer de la estadía del turista y/o visitante más placentera el viaje o desplazamiento) sea efectiva la ruta o circuito turístico.

Este enfoque es muy decidor e importante para consolidar esta propuesta, que, en las actuales circunstancias, se hace necesario el análisis espacial y de uso de las aplicaciones que 
generan una economía inteligente que a base de oferta y demanda construyen un mercado de intercambio de bienes o servicios, lo que consolida la transformación toyotista del turismo en las actuales circunstancias.

\section{CONCLUSIONES}

Se destaca las infinitas posibilidades de trabajar en este enfoque integral que agrupe a varias disciplinas para consolidar la idea multi y transdisciplinar de las mismas, en base a una acción delimitada de acción y funcionalidad, que abarque varias posibilidades reales de argumento desde el diseño, TIC, multimedia, turismo, economía entre otras.

La aplicación de este tipo de herramientas se ampara desde la consolidación del territorio inteligente el cual pasa a ser visualizado y gestionado de manera inteligente por las diferentes instancias. Es importante por lo tanto pasar a la acción local para consolidar la propuesta del modelo de gestión del Destino Turístico Inteligente (Cuenca Destino Turístico Inteligente, 2020).

Esta investigación, pretende sistematizar y homogeneizar el levantamiento de la información sobre atractivos turísticos de la zona de estudio, en este sentido, proponer que sea utilizado en la elaboración de planes de desarrollo sustentables de turismo, planificación y proyectos turísticos, así como por todas las personas e instituciones públicas y privadas, como forma de apoyar la innovación tecnológica y su utilización en el turismo. 


\section{REFERENCIAS BIBLIOGRÁFICAS}

Aguayo, A.; Guevara, A.; Rossi, C.; Caro, J. L. \& Leiva, J. L. (2010). "Sistema integrado de gestión de destinos". Congreso Turitec, Málaga.

Blanco, J. (2015). Libro blanco de los destinos turísticos inteligentes: estrategias y soluciones para fomentar la innovación en el turismo digital. Madrid, Espanha: LID Editorial Empresarial.

\section{Google Scholar}

Blanco Vila, L. (1995). El camino de Santiago. Editorial Complutense. Madrid.

\section{Google Scholar}

Boullon, R. (1995). Planificación del espacio turístico. Editorial Trillas. México.

\section{Google Scholar}

Buhalis, Dimitrios \& Amaranggana, Aditya. (2015). Smart Tourism Destinations Enhancing Tourism Experience Through Personalisation of Services. Information and communication technologies in tourism 2015. Doi: https://doi.org/10.1007/978-3319-14343-9_28.

Cabezuelo-Lorenzo, F., Bonete-Vizcaíno, F., \& SánchezMartínez, M. (2016). Análisis de la información y documentación científica española sobre el fenómeno de las smart cities, el hábitat de los nativos digitales. Cuadernos de documentación multimedia, 27(1), 102-124.

\section{Google Scholar}

Castro, M. (2010). Visualización de datos del inventario de atractivos turísticos de la ciudad de Cuenca y el Valle del Santa Bárbara. Trabajo final de Master. Cuenca, Ecuador. Universidad del Azuay.

Castro, P. (2008). "Sistema informático para el inventario de atractivos turísticos” en II Congreso de Turismo. Universidad Austral de Chile. Valdivia, Chile.
Castro, P. (2016). Propuesta metodológica para el inventario de atractivos turísticos. Universidad de La Habana, Trabajo referativo de Turismo. La Habana.

Castro, P. Vasques, Y. Salgado, E. (2020). Propuesta de un modelo de gestión para el Destino Turístico Inteligente (DTI), para la ciudad de Cuenca, Ecuador. Tech Converge, Revista Científica. Volumen No.2. ISSN: 2661-6858.

Castro, P. (2017). "Definición de un modelo operativo para el destino turístico inteligente de Cuenca, Ecuador" en I Congreso CIVITIC 2017. Universidad del Azuay. Cuenca, Ecuador.

Castro, P. Vásquez, Y., Salgado, E. (2019). "Modelo de gestión para el destino turístico inteligente, para Cuenca, Ecuador.

Comunidad Valenciana (2014). Manual de destinos turísticos inteligentes. Valencia, España.

Consejo de Ministros de España (2015). Plan Nacional Integral de Turismo 2012-2015. Recuperado de https:/turismo.gob.es/es-es/servicios/Documents/PlanNacional-Integral-Turismo-2012-2015.pdf

Cuenca Destino Turístico Inteligente. (2020). Recuperado de http//: www.smarturcuenca.com

Fernández, M. Cuadrado, R. (2014). El impacto de las nuevas tecnologías en el sector turístico: aplicación de la realidad aumentada al turismo cultural. Cultura, desarrollo y nuevas tecnologías: VII jornadas de investigación en turismo (2014), p 317-333. ISBN/ISSN 978-84-942296-2-6.

Fernández, M. (2016). Descifrar las Smart Cities: ¿Qué queremos decir cuando hablamos de Smart Cities? CALIGRAMA.

Google Scholar

Guzmán, T. J. L. G., \& Cañizares, S. M. S. (2009). Turismo comunitario y generación de riqueza en países en vías de desarrollo. Un estudio de caso en El 
Salvador. REVESCO. Revista de Estudios Cooperativos, (99), 85-103.

Google Scholar

Lansdale, D. Castro, P. Guerrero, C. (2020). Smart Destinations: Harnessing Technology to Promote Transformation and Sustainability Through Ecotourism in Emerging Market Communities. (C) Springer Nature Switzerland AG 2020.W. Leal Filho et al. (eds.), Zero Hunger, Encyclopedia of the UN Sustainable Development Goals. https://doi.org/10.1007/978-3-319-69626-3_94-1.

López de Lucio, R. (2011). Lecturas recomendadas: Alfonso Vegara y Juan Luis de las Rivas, "Territorios inteligentes. Nuevos horizontes del urbanismo". Urban, 0(12), 171-172. Recuperado de http://polired.upm.es/index.php/urban/article/vie $\mathrm{w} / 490$.

Valles, D. M. (1999). Las Tecnologías de la Información y el Turismo. Estudios turísticos, (142), 3-24.

\section{Google Scholar}

SEGITTUR, (2015). Los destinos turísticos inteligentes. Madrid.

Vila, L. B. (1995). El camino de Santiago. Editorial Complutense.

Google Scholar

Zimmer, P, Grassmann S. (1996). Evaluar el potencial turístico de un territorio. Observatorio Europeo. Leader. Extremadura.

Google Scholar

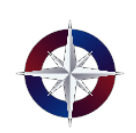

